\title{
Polimorfismo del gen de la catecol-0-metiltransferasa (COMT) en gestantes con restricción del crecimiento intrauterino (RCIU)
}

\author{
José Pacheco, Doris Huerta, Oscar Acosta, Santiago Cabrera
}

Facultad de Medicina, UNMSM

Objetivos: Establecer la asociación entre el polimorfismo Vall58Met catecol-0-metiltransferasa (COMT) y la RCIU.

Diseño: Estudio relacional, observacional, tipo caso-control.

Institución: Facultad de Medicina, UNMSM.

Participantes: Gestantes sin y con RCIU.

Intervenciones: Se obtuvo 81 muestras de sangre para genotipaje del gen COMT; 55 (67,9\%) correspondieron a gestantes $\sin \mathrm{RCIU}$ (controles) y $26(32,1 \%)$ a madres de hijos con RCIU. Las gestantes firmaron consentimiento informado.

Principales medidas de resultados: Frecuencias de los genotipos (Met/Met, Val/Met y Val/Val) y alelos (Val y Met), asociación con la RCIU.

Resultados: Las distribuciones de los genotipos en los grupos con y sin RCIU estuvieron de acuerdo a la hipótesis del equilibrio de Hardy-Weinberg. Al relacionar los genotipos COMT Val/Met con la condición de RCIU, no se apreció diferencias significativas $\left(X_{2}=1.8057, \mathrm{gl}=2, \mathrm{p}=0,4054\right)$. Similar resultado se obtuvo cuando se relacionó los alelos COMT y la RCIU $(\mathrm{X} 2=0.3659, \mathrm{gl}=1, \mathrm{p}=0,5441)$.

Conclusiones: No se ha establecido asociación entre los genotipos y alelos Val/Met en el gen COMT y la RCIU en esta muestra evaluada. Se sugiere ampliar el tamaño de la muestra y seguir investigando el gen COMT, en interacción con otros genes, y su asociación con la RCIU, en la población peruana.

Palabras clave: Restricción de crecimiento intrauterino, fisiopatología, catecol-0-metiltransferasa, catecol estrógenos, polimorfismo Val158Met del gen COMT.

\section{Estudio comparativo de la reactividad serológica a Toxoplasma gondii en pacientes atendidos con diagnóstico de esquizofrenia versus controles}

Luis Marocho, Javier Zegarra, Nelly Almendras, Esther Valencia, Giuliana Romero, Luis Solano, Marcos Ñavincopa, Ana Huamán, Carlos Sevilla, Jorge Chumpitaz

Instituto de Medicina Tropical Daniel A. Carrión, Facultad de Medicina, UNMSM

Objetivos: Evaluar el grado de asociación entre toxoplasmosis y esquizofrenia, comparando el nivel de anticuerpos antitoxoplasma en sangre de pacientes con diagnóstico de esquizofrenia con el nivel de anticuerpos contra dicho agente, en pacientes categorizados como controles.

Diseño: Descriptivo, transversal y de casos y controles.

Institución: Instituto de Medicina Tropical Daniel A. Carrión, Facultad de Medicina, UNMSM.

Participantes: Pacientes con diagnóstico de esquizofrenia (F20), pacientes con otros trastornos mentales.

Intervenciones: Se tomó datos y muestra de sangre durante el año 2011 a 114 pacientes del Hospital Hermilio Valdizán, INCN y Hospital 2 de Mayo, que fueron clasificados según el CIE en F20 (esquizofrenia) y otras enfermedades mentales. Se realizó la titulación de Acanti toxoplasma por el método de Elisa.

Principales medidas de resultados: Determinación serológica de anticuerpos antitoxoplasma en pacientes esquizofrénicos.

Resultados: En pacientes F20 se demostró reactividad serológica en 20 (69\%), en otras enfermedades mentales 9 $(50 \%) ; 15$ de 20 pacientes F20 presentaron valores superiores al doble del valor mínimo (10 MU/mL), es decir, $75 \%(20$ a $160 \mathrm{UI} / \mathrm{mL}$ ). En el grupo de otras enfermedades mentales, no se encontró valores tan altos como los F20.

Conclusiones: Se estableció asociación estadística entre la reactividad serológica a toxoplasmosis y el diagnóstico de esquizofrenia en el grupo estudiado. Los valores de reactividad en pacientes F20 indicaron más del doble de los valores mínimos establecidos como reactivos.

Palabras clave: Toxoplasmosis, esquizofrenia, Acanti toxoplasma. 